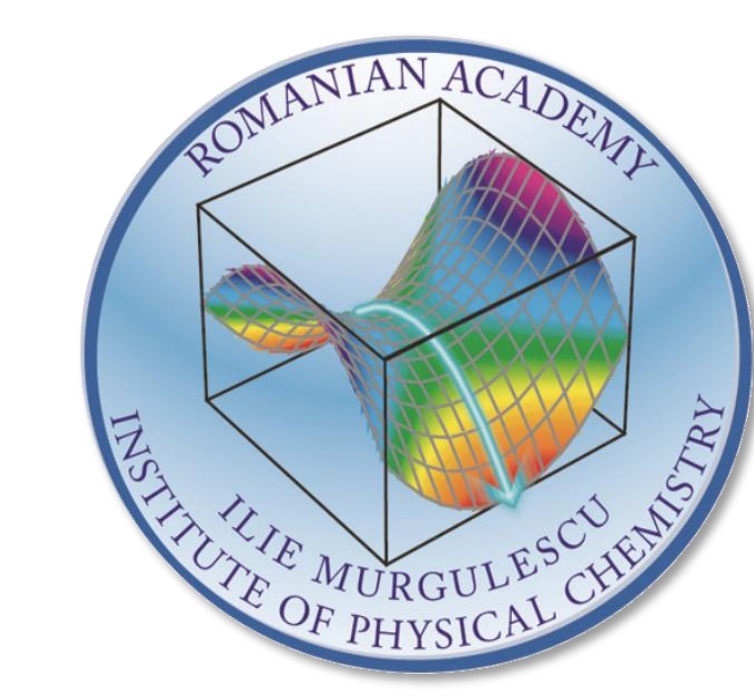

\title{
Preliminary in vitro evaluation of some unsymmetrical porphyrins using human MCF-7 breast tumor cells
}

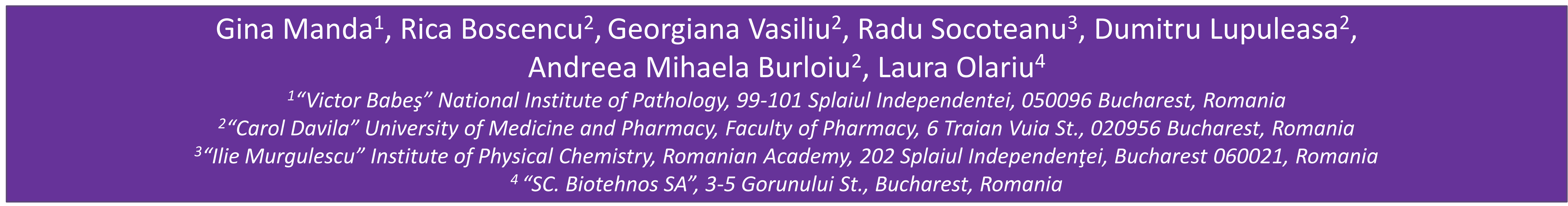

\section{Abstract}

Tetrapyrrolic compounds such as porphyrins and metalloporphyrins are highly interesting for pharmaceutical chemistry designs considering their good biocompatibility and therapeuthic potential [1-3]. The aim of the present work was a preliminary in vitro evaluation of thrree unsymmetrical porphyrins and the corresponding symmetrical structures, as potential candidates for the photodynamic therapy of malignant tumors: 5 - $(2$ - hydroxyphenyl) - 10, 15, 20 - tris - (4 acetoxy - 3 - methoxyphenyl) porphyrin, Zn(II) - 5 - (2 - hydroxyphenyl) - 10, 15, 20 - tris - (4 - acetoxy - 3 - methoxyphenyl) porphyrin, Cu(II) - 5 - (2 - hydroxyphenyl) 10, 15, 20 - tris - (4 - acetoxy - 3 - methoxyphenyl) porphyrin, 5, 10, 15, 20 - meso -tetrakis - (4 - acetoxy - 3 - methoxyphenyl) porphyrin, Zn(II) - 5, 10, 15, 20 - meso tetrakis - (4 - acetoxy - 3 - methoxyphenyl) porphyrin, Cu(II) - 5, 10, 15, 20 - meso -tetrakis - (4 - acetoxy - 3 - methoxyphenyl) porphyrin [4, 5]. The biocompatibility of these compounds was assessed in terms of their in vitro effect on the viability and proliferation of breast human carcinoma MCF-7 cells and human norma peripheral blood mononuclear cells (PBMCS). Results indicated that the new assymetric and symetric porphyrins were non-toxic against tumor MCF-7 cells and PBMCs in the concentration range 0.2-2 $\mu \mathrm{M}$, making them valuable candidates for further development as photosensitizers for PDT in tumors. Moreover, assymetric compounds tended to restore the response of normal and tumor cells affected by DMSO, whyle the symetric compounds were less active in this respect.

Keywords: unsymmetrical porphyrins, human breast carcinoma MCF-7 cell line, human peripheral blood mononuclear cells

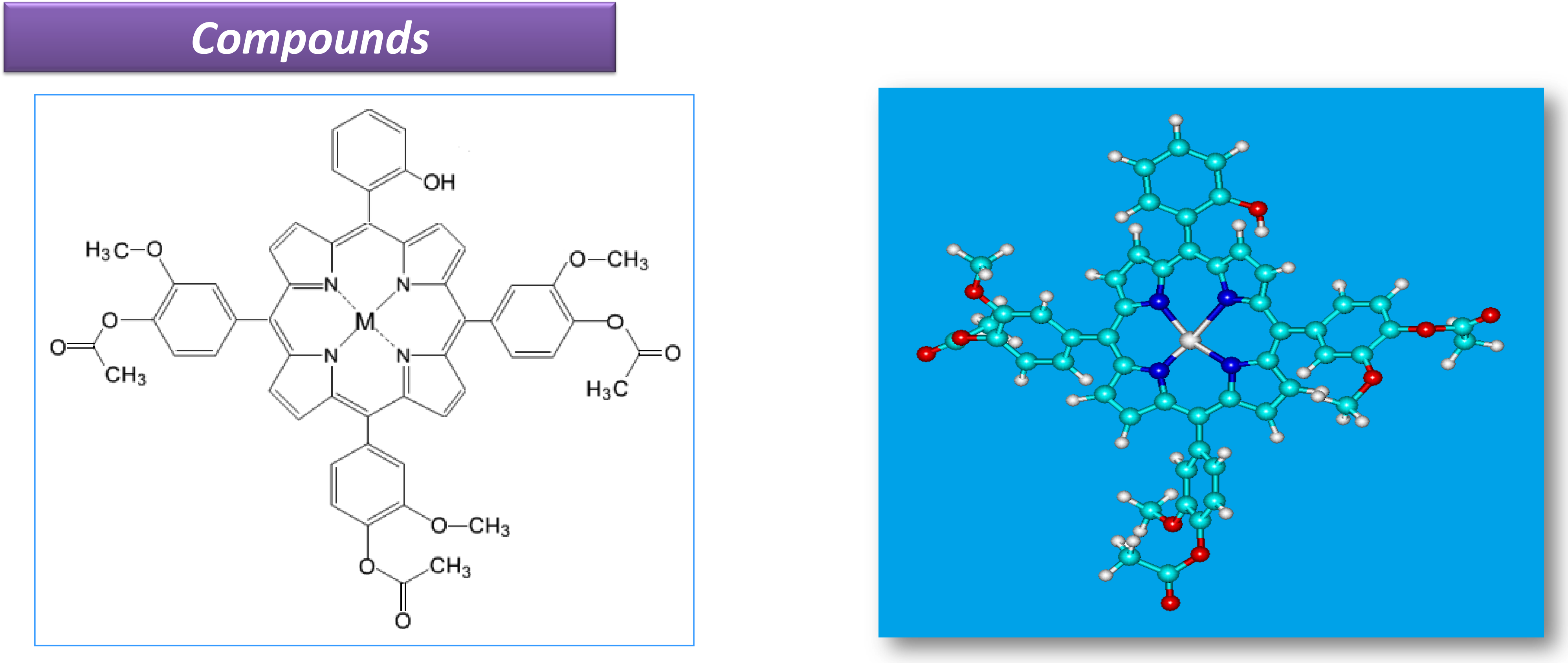

Figure 1. General structures (classic and in silico optimized) of the unsymmetrical porphyrins used in this study 5-(2-hydroxyphenyl)-10, 15, 20-tris-(4-acetoxy-3-methoxyphenyl)porphyrin, $\mathrm{M}=2 \mathrm{H}$, (TMAPOHo)

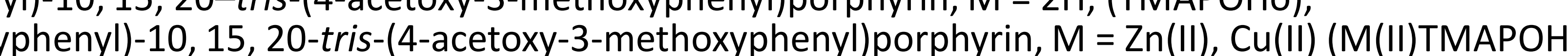

\section{Results}

Figure 3. The effect exerted in vitro by assymetric (a) and symetric (b) porphyrins, as well as by the solvent (DMSO) on MTS reduction by human breast carcinoma MCF-7 cells. Results are presented as mean \pm SEM of triplicate samples.

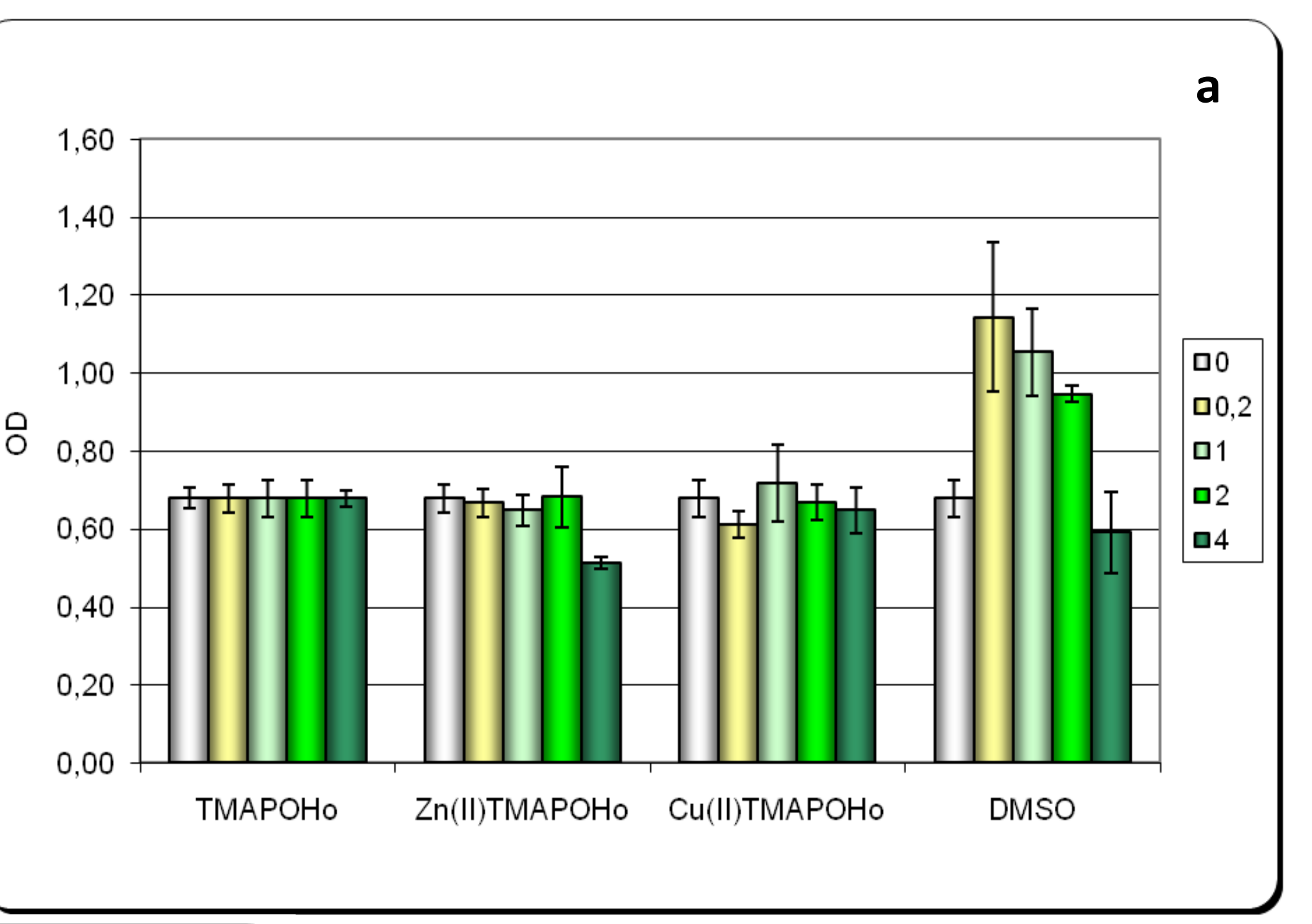

Figure 4. The effect exerted in vitro by the assymetric and symetric porphyrinic compounds (2 $\mu \mathrm{M}$ concentration) on MTS reduction by PBMCs (c) and human breast carcinoma MCF-7 cells (d). Results are presented as mean \pm SEM of triplicate samples.
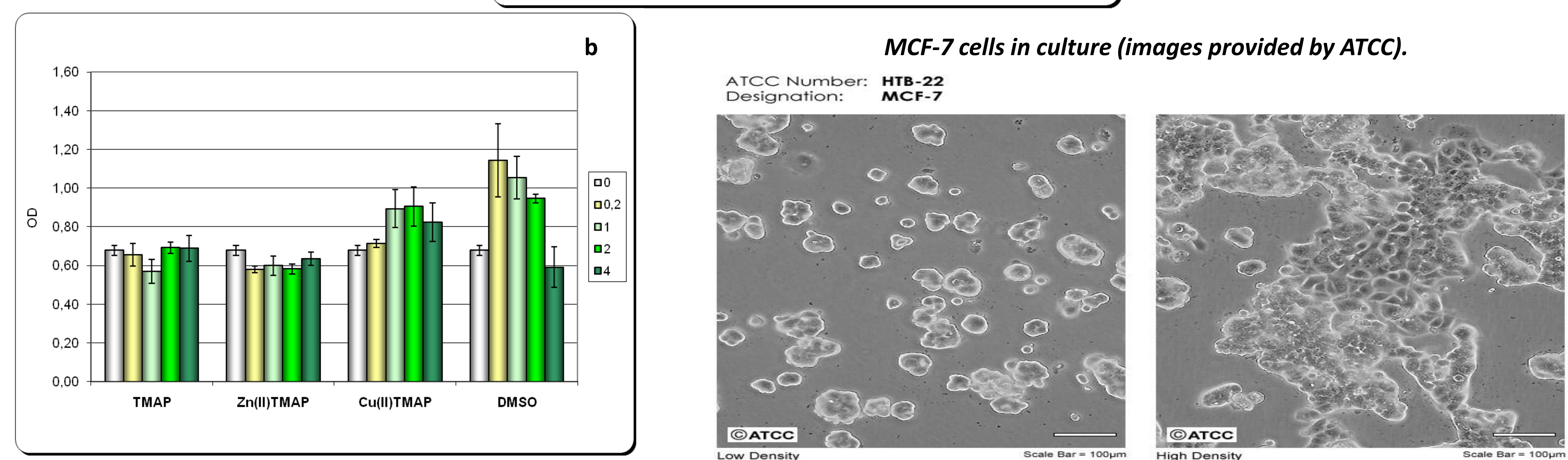

\section{Conclusions}

In this study we evaluated three new unsymmetrical porphyrins and corresponding compounds with symmetrical structure, from the point of view of their effects exerted in vitro on MTS reduction by human breast carcinoma MCF-7 cells and human PBMCs. Results revealed that the new assymetric and symetric porphyrins were non-toxic against tumor MCF-7 cells and PBMCs in the concentration range $0.2-2 \mu M$, making them valuable candidates for further development as photosensitizers for PDT of tumors. Moreover, the investigated assymetric porphyrins tended to restore the response of normal and tumor cells affected by DMSO, while symetric compounds had a lower modulatory action.

\section{References}

. Zhang J., Jiang C., Longo J. P. F., Azevedo R. B., Zhang H., Muehlmann L. A., Acta Pharmaceutica Sinica B, 2018, 8, 137-146.

2. Sandland J., Malatesti N., Boyle R., Photodiagnosis and Photodynamic Therapy, 2018, 23, 281-294.

3. Sandland J. and Boyle R. W., Bioconjugate Chem., 2019, 30, 975-993.

4. Boscencu R., Socoteanu R., Vasiliu G., Nacea V., Rev. Chim., 2014, 65, 888-891.

5. Vasiliu G., Boscencu R., Socoteanu R., Nacea V., Rev. Chim., 2014, 65, 998-1001.

Acknowledgments: This study was supported by ERA NET projects: Biomark and Nanother of the Romanian Ministry of Education and Research 